Forschende

Komplementärmedizin un

Klassische Naturheilkunde

\title{
Zirkulär statt hierarchisch ${ }^{1}$
}

Kreise und Rundungen faszinieren uns Menschen und verwirren uns zugleich. Wir drehen uns im Tanz und freuen uns, gleichzeitig wird uns womöglich schwindlig. Das Karussell, das sich rasch dreht, erinnert uns aus Kindertagen an die Faszination und den Schrecken der Fliehkraft. Wir denken an die Verwunderung zurück, die uns beschlich als wir sahen, wie ein Eimer voll Wasser, auf den Kopf gestellt, sich leert, im Kreis geschwungen aber seinen Inhalt behält. Heute noch sind Menschen fasziniert von den Steinkreisen aus prähistorischen Zeiten. Der Kreis fasziniert, weil er Unendlichkeit suggeriert, Perfektion und Gleichheit. In seiner dreidimensionalen Form, der Kugel, sogar noch mehr als in der zweidimensionalen. Ein Kreis oder eine Kugel haben weder oben noch unten, keine gute und schlechte, keine lange und keine kurze Seite.

Auch das Dreieck und sein dreidimensionales Äquivalent, die Pyramide, begeistern uns. Von den Pyramidenbauten der Ägypter bis hin zum Trinitätssymbol des Gottesauges war das Dreieck bis tief in die Neuzeit hinein das ikonographische Signum des menschlichen Geistes. Viele Zahlenspekulationen, von Pythagoras bis Plotin und Augustinus belegen die Bedeutung der Dreizahl, die im Trinitätsgedanken der christlichen Ära ihre Krönung fand.

Die politisch-soziale Entsprechung des Dreiecks und der Pyramide ist die Hierarchie, die des Kreises das gemeinschaftliche Diskutieren und die soziale Verhandlung im Konsens. Es dürfte nicht von ungefähr kommen, dass die Erbauung der ersten Pyramiden und die hierarchische Durchstrukturierung eines Staatswesens Hand in Hand gingen, wenn nicht sogar die sozial-politische Hierarchisierung Voraussetzung für den Bau von Pyramiden war. Das biblische Mythologem vom Turmbau zu Babel - erinnern wir uns: die ersten pyramidenartigen Bauten im Mittleren Osten waren die astronomischen Türme der Sumerer - sagt uns auch, welchen Preis dieses Vorhaben forderte: den Verlust der allgemeinen Verständigung und Kommunikation. Die Sprachverwirrung ist Folge

\footnotetext{
${ }^{1}$ Werner W. Wittmann zum 60. Geburtstag ins Stammbuch geschrieben.
}

des Turmbaus. Die konsequente Hierarchisierung wurde vom spätantiken römischen und byzantinischen Staatswesen weiterentwickelt, von dort auf die Struktur der mächtigen Kirche übertragen, und von dort auf die politischen Instanzen des Abendlandes. Die Demokratisierung der Gesellschaft im Zuge der politischen Aufklärung und Revolutionen darf nicht darüber hinwegtäuschen, dass wir immer noch hierarchisch denken. Man gibt dem Präsidenten oder dem Kanzler Schuld, wenn etwas schief läuft, und wägt sich im Irrglauben, dass das Absetzen von unliebsamen Präsidenten und die Abwahl von inkompetenten Kanzlern die Situation ändern wird. Hierarchie hat auch manches für sich: Nicht alle müssen sich um alles kümmern, sondern man delegiert, nach oben und unten. Schliesslich ist auch unser Körper in Grenzen hierarchisch aufgebaut: Viele immer gleiche Zellbestandteile bilden in unterschiedlichen Formationen ganz unterschiedliche Zellen, die wieder ganz unterschiedliche Organe formen, die alle zusammen einen Organismus bilden, an dessen operativer Spitze so etwas wie eine Steuerungseinheit, unser Ich, seinen Platz hat. So mindestens sehen wir es meistenteils, und in mancher Hinsicht stimmt dies auch.

Jean Gebser, der wenig rezipierte und doch immens bedeutende Kulturanthropologe, hat in seinem Hauptwerk «Ursprung und Gegenwart» [1, 2] darauf hingewiesen, dass das Dreieck und die Dreiecksstruktur das Signet der sog. mentalen Phase sind, jener Phase, in dem das reflexive Ichbewusstsein des Menschen zu seiner Blüte gelangt. Gleichzeitig, sagt Gebser, zeigt sich in unserer Zeit an verschiedenen Orten eine neu aufbrechende Struktur des Bewusstseins, die er die «integrale» nennt. Ihr sei, im Gegensatz zur mentalen, eine vertieftere und partizipatorischere Art eigen, sich mit der Welt zu verbinden. Genauer gesagt sei eigentlich die Realisation von Verbundenheit einer der Kernpunkte eines integralen Bewusstseins. Möge nun Gebser Recht haben oder nicht, interessanterweise macht er anhand verschiedener Beispiele aus der Kunst die Kugel als das Ikonogramm dieser Phase aus. Der Kreis in seiner dreidimensionalen Form zeigt sich uns also als Ergänzung, ja sogar als Nachfolger des Dreiecks, Partizipation als Nachfolgerin hierarchischer Herrschaftsstruktur.

\begin{tabular}{ll}
\hline KARGER & @ 2004 S. Karger GmbH, Freiburg \\
Fax +497614520714 & Accessible online at: \\
$\begin{array}{l}\text { E-mail Information@Karger.de } \\
\text { www.karger.com }\end{array}$ & www.karger.com/fkm
\end{tabular}

PD Dr. Dr. phil. Harald Walach

Klinikum der Universität

Institut für Umweltmedizin und Krankenhaushygiene

Hugstetter Str. 55

D-79106 Freiburg

Tel. +49 761 270-5497/98, Fax -7224. E-mail walach@ukl.uni-freiburg.de 
Die Hierarchisierung ist das Kennzeichen des methodischen Credos einer Reihe von medizinischen Forschern, vielleicht sogar der Mehrheit. An der Spitze der Pyramide steht, seiner Validität wegen, die randomisierte, kontrollierte Studie, idealerweise sogar plazebokontrolliert und verblindet. An der Basis und dazwischen andere Methoden, wie Fallserien, Dokumentations- und Kohortenstudien oder pragmatische randomisierte Studien. Und so wie der Papst, der König, der Kaiser, der Bundeskanzler, der Präsident, der Konzernchef oder der Chefarzt am Ende so entscheidet, wie er es für richtig hält, vielleicht sogar gegen die Meinung der Basis, so entscheidet bei Fragen der Wirksamkeit am Ende die gesammelte Beweislast der randomisierten Studien, möglicherweise sogar gegen die Daten an der Basis und dazwischen.

Diesem hierarchischen Modell will ich das zirkuläre gegenüberstellen. Es ist der Sache nach verschiedentlich dargestellt worden [3-5] und wird demnächst auch noch detaillierter nachzulesen sein [6]. Die Grundidee lautet: Es gibt keine Hierarchie von Methoden, die implizit und explizit so genannte «gute» von «schlechten» Methoden unterscheidet, «validere» von «weniger validen», sondern es gibt nur angemessene Methoden für bestimmte Fragen und die organisatorischtechnisch gute Implementation von Methoden oder stümperhafte Anwendung. Eine organisatorisch-technisch gut eingefädelte Outcomes-Studie mit grosser Fallzahl sagt noch immer mehr aus als eine schlecht gelaufene, mit Rekrutierungsmängeln behaftete, wenig repräsentative randomisierte Studie. Während das hierarchische Modell suggeriert: einer hat Recht, einer entscheidet, einer weiss es besser, eine Methode schlägt alle anderen, vermittelt das zirkuläre: jede Methode zu ihrer Zeit, keine ist besser als die andere, alle sind wichtig, um ein vollständiges Bild zu erhalten. Während das hierarchische Modell die Information verdichtet, gleichsam in einen Trichter zwängt und am Ende idealerweise mit einem Stück Information vor die Öffentlichkeit tritt, nämlich mit der vermeintlich wahren Effektgrösse einer Intervention, legt das zirkuläre Modell nahe: Je nach Blickwinkel, je nach Erkenntnisinteresse, je nach Fragestellung zeigt sich ein anderes Bild.
Alle Perspektiven sind für sich genommen in gleicher Weise gültig und erst alle gemeinsam ergeben ein vollständiges Bild. Während die Pyramide eine Basis hat und eine prominente Spitze, hat der Kreis keine vorgezogene oder vorzügliche Seite. Alle Seiten, alle Zugänge, alle Anfangspunkte sind gleichberechtigt. «Every end is a beginning, every beginning is an end», dichtete T.S. Eliot in seinen «Four Quartets», und verdichtete damit die Ikonographie der Kreisstruktur meisterhaft. Der Kreis kann an jeder Stelle betreten werden und an jeder Stelle verlassen. Wo man ihn betritt, hängt von der Fragestellung ab, und wo man ihn verlässt vom Zeitpunkt an dem man meint, genug zu wissen. Das Kriterium des Belegs von Wirksamkeit, des Beweises von Effektivität ist nicht das Urteil, das randomisierte Studien sprechen, sondern die Konvergenz von Daten aus unterschiedlichen Kontexten, Designs, Fragestellungen und Randbedingungen. Möglicherweise konvergieren sie zu einem klaren Bild. Manchmal scheint dies so zu sein [7], manchmal aber auch nicht. Vielleicht wird in manchen Fällen die Situation schillernd bleiben. Verfahren mögen unter bestimmten Randbedingungen sehr wirksam sein - etwa wenn Menschen sich bewusst für ihre Anwendung entscheiden und sich etwas davon erhoffen -, unter anderen Bedingungen unbrauchbar - etwa dann, wenn sie einem Skeptiker oder Indifferenten aufgezwungen werden. Auch solche Randbedingungen zu kennen gehört zum Gesamtbild.

Es wird Zeit, dass wir uns von idealisierenden Fiktionen verabschieden: In der Komplementärmedizin im Speziellen, aber auch in der Medizin im Allgemeinen leiden wir an der Fiktion, es gäbe so etwas wie die Wirksamkeit einer Intervention, unabhängig von Kontext, Anwender und Empfänger. Sie ist ein Resultat der methodischen Fiktion, es gäbe so etwas wie die Methode der Forschung schlechthin. Diese wiederum ist ein Resultat hierarchischer Denk-, Arbeits-, Lebens- und Bewusstseinsstrukturen. Es wird Zeit, dass wir ein zirkuläres Modell leben und entwickeln, methodisch, in unseren Gesellschaftsund Arbeitsstrukturen, und nicht zuletzt in unserem Bewusstsein. Vielleicht dort zuerst - alles andere ergibt sich dann.

H. Walach, Freiburg i. Br.

\section{Literatur}

1 Gebser J: Ursprung und Gegenwart. Zweiter Band: Die Manifestationen der aperspektivischen Welt. Versuch einer Konkretion des Geistigen. Stuttgart, Deutsche Verlagsanstalt, 1953.

2 Gebser J: Ursprung und Gegenwart. Erster Band: Die Fundamente der aperspektivischen Welt. Beitrag zu einer Geschichte der Bewusstwerdung. Stuttgart, Deutsche Verlagsanstalt, 1949.
3 Lewith G, Walach H, Jonas WB: Balanced research strategies for complementary and alternative medicine; in Lewith G, Jonas WB, Walach H (eds): Clinical Research in Complementary Therapies: Principles, Problems, and Solutions. London, Churchill Livingston, 2002, pp 3-27.

4 Walach H, Jonas WB, Lewith G: The role of outcomes research in evaluating complementary and alternative medicine. Ibid. ref 3, pp 29-45.
5 Wittmann WW, Walach H: Evaluating complementary medicine: Lessons to be learned from evaluation research. Ibid. ref 3, pp 93-108.

6 Walach H: Zirkulär statt hierarchisch: Methodische Prinzipien bei der Evaluation von Komplementärmedizin und anderswo. Informatik Biometrie Epidemiol Med Biol 2004 (im Druck).

7 Concato J, Shah N, Horwitz RI: Randomized, controlled trials, observational studies, and the hierarchy of research designs. N Engl J Med 2000;342: 1887-1892. 